\title{
Bubbly-ice densification in ice sheets: I. Theory
}

\author{
Andrey N. Salamatin, ${ }^{1}$ Vladimir Ya Lipenkov, ${ }^{2}$ Paul Duval $^{3}$ \\ ${ }^{1}$ Kazan State University, Kazan 420008 , Russia \\ ${ }^{2}$ Arctic and Antarctic Research Institute, St. Petersburg 199397, Russia \\ ${ }^{3}$ Laboratoire de Glaciologie et de l'Environnement, 38402 Saint-Martin-d'Hères Cedex, France
}

\begin{abstract}
Dry snow on the surface of polar ice ice sheets is first densified and metamorphosed to produce firn. Bubbly ice is the next stage of the transformation process which takes place below the depth of pore closure. This stage extends to the transition zone where, due to high pressures and low temperatures, air trapped in bubbles and ice begins to form the mixed air clathrate hydrates, while the gas phase progressively disappears. Here we develop a model of bubbly-ice rheology and ice-sheet dynamics taking into account glacier-ice compressibility. The interaction between hydrostatic compression of air bubbles, deviatoric (uniaxial) compressive deformation of the ice matrix and global deformations of the glacier body is considered. The ice-matrix pressure and the absoluteload pressure are distinguished. Similarity theory and scale analysis are used to examine the resultant mathematical model of bubbly-ice densification. The initial rate of bubble compression in ice sheets appears to be relatively high, so that the pressure (density) relaxation process takes place only $150-200 \mathrm{~m}$ in depth (below pore close-off) to reach its asymptotic phase, wherein the minimal drop between bubble and ice pressures is governed by the rate of loading (ice accumulation). This makes it possible to consider densification under stationary (present-day) conditions of ice formation as a special case of primary interest. The computational tests performed with the model indicate that both ice-porosity and bubble-pressure profiles in ice sheets are sensitive to variations of the rheological parameters of pure ice. However, only the bubble-pressure profile distinguishes between the rheological properties at low and high stresses. The porosity profile at the asymptotic phase is mostly determined by the air content in the ice. In the companion paper (Lipenkov and others, 1997), we apply the model to experimental data from polar ice cores and deduce, through an inverse procedure, the rheological properties of pure ice as well as the mean air content in Holocene and glacial ice sediments at Vostok Station (Antarctica).
\end{abstract}

\section{INTRODUCTION}

The formation of glacier ice in the melt-free zone of polar ice sheets results from a complex process of densification of dry snow deposited on the surface and can be presented as a sequence of five stages. For example, at Vostok Station this process has been described by Salamatin and others (1985) and Lipenkov (1989). In the initial stage, the densification of snow is mainly a structural re-arrangement and sintering process due to ice-grain surface forces, the thermal gradient and ice load. Snow has a minimal volume concentration of closed (isolated) pores and is present down to a density of about $0.55 \mathrm{Mg} \mathrm{m}^{-3}$. The second stage is firn. It is characterized by viscoplastic deformation of ice grains under increasing overburden pressure. When all pores become closed, usually at $50-120 \mathrm{~m}$ below the surface, the atmospheric air is trapped in the form of bubbles, and thus the third stage, defined as bubbly ice, comes into being. Further densification of sinking bubbly-ice sediments is essentially a relaxation process corresponding to air-bubble constriction in a plastically deforming polycrystalline ice matrix driven by the pressure drop between the two phases. At a depth of $500-1000 \mathrm{~m}$ (depending on temperature) the bubble pres- sure becomes sufficient for the formation of the mixed air clathrate hydrates (Miller, 1969). Below this depth, the twophase bubbly ice changes into a three-phase aggregate, which is the main attribute of the fourth stage, where air bubbles and air-hydrate crystals coexist in the ice matrix. The gas phase progressively disappears with depth in the transition zone that may be half to twice as thick as the bubbly-ice stratum. Below the lower boundary of the transition zone, the two-phase aggregate (distinguished as the fifth stage) consisting of ice and hydrates is present down to the bottom of the ice sheet. The three phase transformations of glacier ice after pore close-off involve different mechanisms and rates of densification, so they must be distinguished (Lipenkov, 1989). The densification process within the bubbly-ice stratum considered below is of special interest because it is suitable for studying the rheological properties of ice at relatively low stresses. Also, the density distribution within this stratum is presumed to record past variations of initial ice porosity and bubble pressure at pore close-off. Modelling the bubbly-ice densification is still considered to be a necessary step in the theoretical study of air-hydrate formation in ice sheets.

The first quantitative descriptions of the densification of 
bubbly glacier ice under overburden pressure were given by Langway (1958), Bader (1965), Gow (1968), Shumskiy (1969) and Nakawo and Narita (1985) on the basis of phenomenological approaches or simply as appropriate approximations of experimental data. Salamatin and others (1985) employed a more rigorous averaging procedure (Bensoussan and others, 1978) to develop in general the same densification model as the one constructed within the framework of the cell-structure approximation by Wilkinson and Ashby (1975) for simulating ceramics production processes. This model was applied to deduce the parameters of the power creep law of pure ice from porosity profiles measured along five deep ice cores retrieved in Antarctica and Greenland. Later, the inferred rheological parameters of ice were used by Lipenkov and others (1989b) for simulating climatically induced signals in porosity (density) profiles in ice sheets. Using the same theoretical approach, Lipenkov and Salamatin (1989) also studied the volume relaxation of the ice cores recovered at Vostok Station. Results of the investigations carried out in this field in 1982-89 have been reviewed by Salamatin and Lipenkov (1993).

The mathematical models mentioned above essentially use (a) a power creep law for ice and consider (b) bubblyice densification as a process of hydrostatic compression at (c) the averaged pressure in the ice matrix equal to the absolute load pressure. All of these assumptions are somewhat limiting. First, Pimienta (1987) and Pimienta and Duval (1989) have shown that asymptotically, as the bubble-ice pressure drop decreases, the rheological behavior of ice becomes linear viscous. Although this is not revealed in ice-porosity prediction, it changes the estimate of air-bubble pressure at large depths. Secondly, due to the lateral constraints, the deformation of the ice matrix in the bubbly-ice densification process is a composition of hydrostatic and deviatoric uniaxial (vertical) compression of comparable strain rates. Thirdly, the deformation of bubbly ice on the micro-scale of a single bubble is also subjected to additional "external" deviatoric strains induced by the global macroscale movement of the ice sheet. To account for these peculiarities, special research has been carried out to derive general rheological relations governing the creep flow of bubbly ice and its expansion (compression) in pressure relaxation processes (Salamatin and Duval, 1997). The kinematic interaction of ice-sheet flow with compressibility effects has also been considered (Salamatin, 1991). In addition, a comprehensive experimental study of bubbly-ice densification has recently been undertaken on the Vostok ice core. The new data set obtained includes for the first time direct measurements of bubble pressure in unrelaxed ice and enables theoretical models to be more precisely validated by experimental data. Thus, we need: (1) to develop a new model in order to overcome the above-mentioned drawbacks by introducing all the recent theoretical results and (2) to revise the model application, taking into account the new experimental data. In accordance with this two-fold purpose, our work will be presented in two parts: I. Theory and II. Applications. In this first paper, we develop an improved mathematical model sufficient for predicting bubbly-ice densification between the close-off level and the airhydrate formation zone; we also conduct a preliminary investigations and sensitivity study of the model. In the companion paper (Lipenkov and others, 1997) (hereafter referred to as paper II) we use the model to interpret the ice-core data.

\section{GENERAL EQUATIONS GOVERNING DENSIFIC- ATION OF BUBBLY ICE}

Since bubbly ice is a two-phase heterogeneous medium from a mechanical point of view, we should distinguish two space scales when modeling the densification processes: (i) a micro-scale associated with a characteristic size of pores and (or) a characteristic distance between neighboring air inclusions, and (ii) a macro-scale corresponding to the typical dimensions of the domain where bubbly-ice densification takes place. Here, we use a "macro-continual" approach to describe the behavior of the air-ice mixture in terms of the macro-scale, averaged characteristics introduced conventionally in the mechanics of multi-phase media:

$c$, ice porosity (air-volume concentration);

$\rho$, density of bubbly ice, $\rho=\rho_{\mathrm{i}}(1-c)$, where $\rho_{\mathrm{i}}$ is the density of pure ice ( $\rho_{\mathrm{i}}$ is assumed to be constant);

$\mathbf{v}$, averaged velocity vector;

$P_{\mathrm{i}}, P_{\mathrm{b}}$, averaged pressure in the ice matrix and the mean pressure in bubbles, respectively;

$\mathbf{T}$, deviator of macro-stresses in the ice matrix.

The mass and momentum balance equations governing the slow creep motion of glacier ice can be written in the form:

$$
\mathrm{d} \rho / \mathrm{d} t+\rho \nabla \cdot \mathbf{v}=0, \quad \nabla P_{\mathrm{i}}=\nabla \cdot \mathbf{T}+\mathbf{g} \rho
$$

where $\mathbf{g}$ is the gravity vector, $t$ is the time and $\nabla$ is the differential nabla operator.

Equations (2.1) are general. Rigorous definitions of the above characteristics and corresponding constitutive relations completing the system in Equations (2.1) may differ from one stage of densification to another. However, in any case they must be based on and deduced from the analysis of deformation processes on the micro-scale level. Such an analysis has been undertaken for bubbly media by Salamatin and Duval (1997) within the framework of homogenization methods for periodic structures (Bensoussan and others, 1978). The results are used below.

First of all, a simple mass-balance (state) equation holds for a specific volume of ideal gas $c /(1-c)$ in a unit mass of ice provided neither phase change nor gas diffusion in the ice matrix takes place:

$$
\frac{\mathrm{d}}{\mathrm{d} t}\left[\frac{c P_{\mathrm{b}}}{T(1-c)}\right]=0
$$

where $T$ is the temperature $(\mathrm{K})$ treated hereafter as a known value. Equation (2.2) means that the quantity in the square brackets is constant along bubbly-ice particle trajectories.

The near-surface bubbly stratum of the ice sheet is composed of polycrystalline isotropic ice (see e.g. Lipenkov and others, 1989a). Therefore, one can write, as in Glen (1955), Budd (1969) and Shumskiy (1969), the following relation between deviatoric micro-stresses $\tau$ and micro-strain rates $\dot{e}$ in the ice matrix:

$$
\tau=2 \eta\left(4 \mathbf{I I}_{\mathrm{e}}\right) \dot{e}
$$

Rheological coefficient $\eta$ is a function of the second invariant of the strain-rate tensor $\mathbf{I I}_{\mathrm{e}}=0.5 \dot{e}: \dot{e}$ and temperature $T$. 
In addition, the macroscopic bubble-compression rate $\omega$ can be defined as:

$$
\omega=-\nabla \cdot \mathbf{v} / c .
$$

Finally, as proposed by Salamatin and Duval (1997) for ice with mono-dispersed spherical bubbles, we have the following rheological relations which determine the phase pressure drop and the deviatoric stresses in bubbly ice in a deformational process:

$P_{\mathrm{i}}-P_{\mathrm{b}}=$

$$
\frac{2}{\sqrt{3}} \int_{2 \omega \omega / \sqrt{3}}^{2 \omega / \sqrt{3}}\left[\eta\left(\xi^{2}+4 \mathbf{I I}_{\mathrm{E}}\right)+4 \kappa \mathbf{I I}_{\mathrm{E}} \eta^{\prime}\left(\xi^{2}+4 \mathbf{I I}_{\mathrm{E}}\right)\right] \mathrm{d} \xi,
$$

and

$$
\begin{aligned}
\mathbf{T}= & 2\left\{\int _ { c } ^ { 1 } \left[\eta\left(\frac{4 c^{2} \omega^{2}}{3 \zeta^{2}}+4 \mathbf{I I}_{\mathrm{E}}\right)\right.\right. \\
& \left.\left.+\kappa \frac{4 c^{2} \omega^{2}}{3 \zeta^{2}} \eta^{\prime}\left(\frac{4 c^{2} \omega^{2}}{3 \zeta^{2}}+4 \mathbf{I I}_{\mathrm{E}}\right)\right] \mathrm{d} \zeta\right\} \dot{\mathbf{E}} .
\end{aligned}
$$

Here $\mathbf{E}$ is the deviator of the macro-strain-rate tensor:

$$
\dot{\mathbf{E}}=(\nabla \mathbf{v})^{\mathrm{s}}-(\nabla \cdot \mathbf{v} / 3) \delta
$$

where $\delta$ is the identity tensor and superscript "s" denotes symmetrization; $\mathbf{I I}_{\mathrm{E}}=0.5 \dot{\mathbf{E}}: \dot{\mathbf{E}} ; \kappa$ is the tuning parameter, which varies within the range $0.4 \leq \kappa \leq 1$ for different approximations of micro-deformations in the bubble vicinity.

Equations (2.1), (2.2) and (2.4)-(2.7) form a complete model of bubbly-ice creep flow, including bubble-pressure (density) relaxation effects. The deviatoric deformations of ice $\left(\mathbf{I I}_{\mathrm{E}}\right)$ and the bubble compression $(\omega)$ are closely interrelated, since both processes are represented equally and symmetrically in the macro-scale rheological Equations (2.5) and (2.6).

Originally, the latter relations were derived for a periodic composition of ice with spherical bubbles. In reality, the shape of air inclusions can be rather irregular, especially in young ice, immediately after pore close-off. Yet, we believe Equations (2.5) and (2.6) remain approximately valid in this case because the air-volume concentration in ice is relatively low $(c<0.1)$ and the deformation of ice-matrix cells each containing a small single bubble does not depend at first order on the bubble shape.

\section{ANALYSIS OF DEFORMATION PROCESSES IN THE UPPER STRATUM OF THE ICE SHEET}

The compressible air bubble-ice medium forms a relatively thin layer in the upper part of ice-sheet thickness. This specific stratum does not noticeably influence the overall movement of the glacier but is involved in complicated deformation processes which are responsible for the distribution of the vertical velocity near the ice-sheet surface (see Fig. la). Here, we consider the kinematic interrelation between the global ice-sheet flow and the densification, as initially looked at by Salamatin (1991).

\section{Interrelation of ice-sheet flow and compressibility effects}

Let $s$ be the distance from the ice divide along a certain flow tube confined between two close flowlines with a relative width $H(s)$, as shown in Figure la. The vertical coordinate is noted as $z$ (the $z$ axis is directed upward); $u, w$ are the corresponding longitudinal and vertical velocities; $l, z_{0}$ are respectively the ice-sheet surface and bed elevation; $b$ is the ice accumulation rate, and $u_{0}, w_{0}$ are respectively, the sliding velocity and melting rate of ice at the glacier bottom.

It is also helpful to introduce the elevation of glacier surface $\left(l^{\prime}\right)$ and the modified coordinate $\left(z^{\prime}\right)$ measured in equivalent of pure ice:

$$
z^{\prime}=z_{0}+\int_{z_{0}}^{z}(1-c) \mathrm{d} z, \quad l^{\prime}=z_{0}+\int_{z_{0}}^{l}(1-c) \mathrm{d} z .
$$

The total volume rate of ice in the tube through its cross-section of unit width is

$$
A(s, t)=\frac{1}{H} \int_{0}^{s}\left(b-w_{0}-\partial l^{\prime} / \partial t\right) H(s) \mathrm{d} s,
$$

the corresponding flow rate due to sliding is $\left(l^{\prime}-z_{0}\right) / u_{0}$, and the relative fraction of the flow rate caused by plastic shear deformations within the glacier body is then defined as

$$
\sigma=1-\left(l^{\prime}-z_{0}\right) u_{0} / A .
$$

The latter ratio varies from 0 (no shear within the glacier) to 1 (no slip in the basal layer). In some cases and, in particular, in the ice-sheet densification modeling this variable is likely to be considered as an adjustable (tuning) parameter.

Closer examination of the general Equations (2.1) for ice-creep motion along a flow tube under conventional boundary-layer (shallow-ice) approximation assumptions allows the profiles for the vertical and longitudinal velocities to be written explicitly with the snow-firn-bubbly-ice compressibility taken into account (Salamatin, 1991):

$$
\begin{aligned}
u= & \frac{A}{l^{\prime}-z_{0}}[1-\sigma(1-G)], \\
w= & -\frac{b}{\left.(1-c)\right|_{\bar{z}=1}}+\frac{\partial l}{\partial t}+\left.u\right|_{\bar{z}=1} \frac{\partial l}{\partial s} \\
& +\int_{0}^{h} c \omega \mathrm{d} h+\frac{h}{H} \frac{\partial}{\partial s}\left(\left.H u\right|_{\bar{z}=1}\right) \\
& +\frac{1-\bar{z}}{H} \frac{\partial}{\partial s}\left[\sigma H A\left(\frac{1-F}{1-\bar{z}}-\left.G\right|_{\bar{z}=1}\right)\right] \\
& +\frac{\sigma A}{l^{\prime}-z_{0}}\left[\bar{z} \frac{\partial l^{\prime}}{\partial s}+(1-\bar{z}) \frac{\partial \bar{z}_{0}}{\partial s}\right]\left(G-\left.G\right|_{z=1}\right) .
\end{aligned}
$$

Here, $h=l-z$ is the depth and $\bar{z}=\left(z^{\prime}-z_{0}\right) /\left(l^{\prime}-z_{0}\right)$, the normalized vertical coordinate. The functions $G(\bar{z})$ and $F(\bar{z})$ describing power-law shear in the glacier body are conventionally presented in an integral form (see e.g. Salamatin, 1991).

The first formula in Equations (3.3) directly represents the longitudinal velocity $u$ as the mean slab-flow velocity and superposed shear component for $\sigma>0$. The structure of the second formula for vertical velocity $w$, although more complicated, is also clear. First terms respectively show contributions of (1) snow accumulation rate, (2) vertical motion of the ice-sheet surface, (3) densification rate of porous ice deposits, and (4) vertical deformation of the ice-sheet thickness in the slab-like flow at the surface horizontal velocity. The last two terms (being proportional to $\sigma$ ) describe the direct impact of the shear strains in the glacier body and 


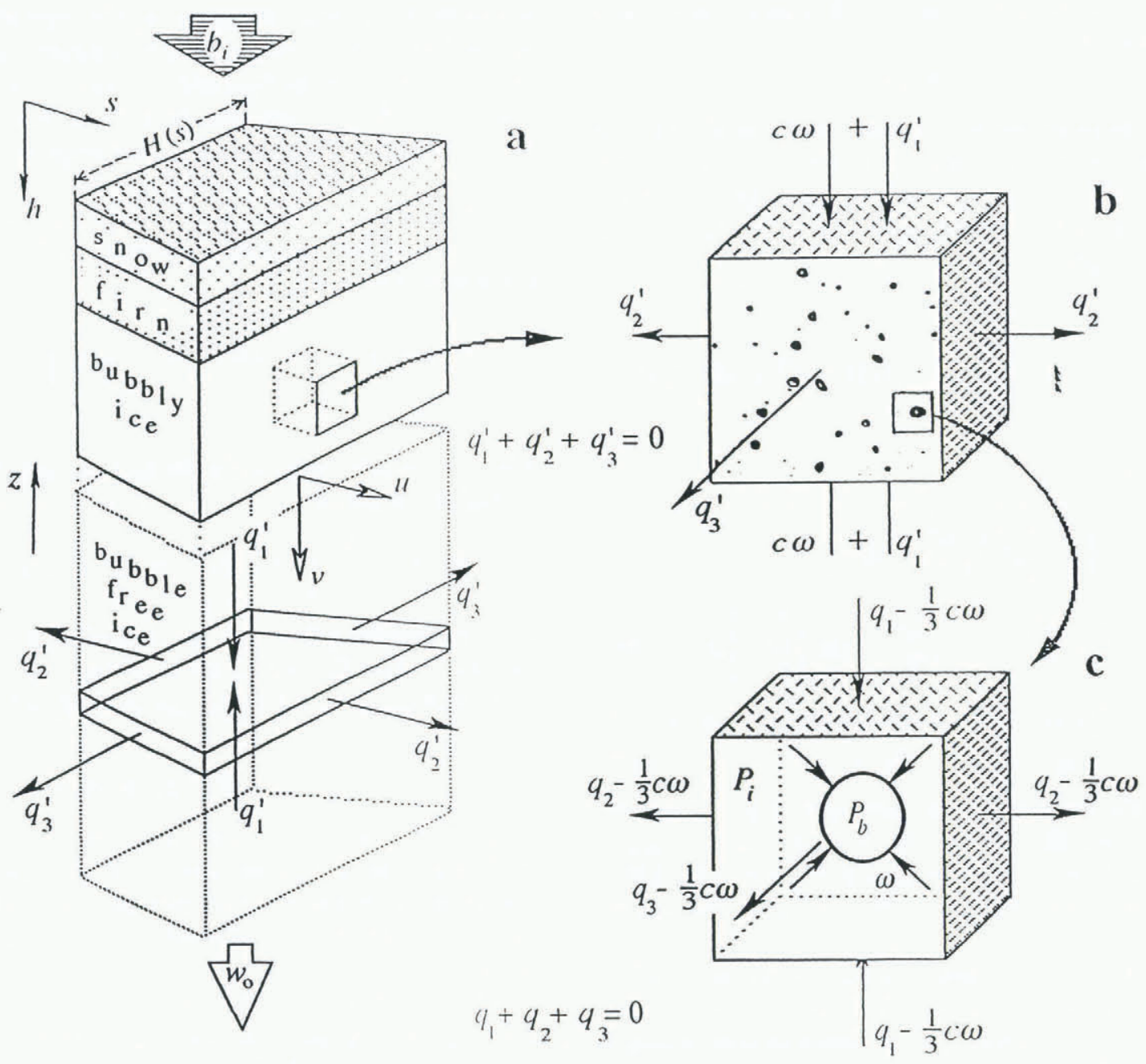

Fig. 1. Different scales of ice deformation to be considered when modeling bubbly-ice densification in ice sheels (see the text for the meaning of the denotations). (a) Fragment of ice-flow tube representing the "external" motion of the ice sheet. (b) Bubbly-ice aggregate or particle (macro-scale). (c) Unit cell containing a single bubble (micro-scale).

the influence of the ice thickness and the bed-relief lateral variations on the vertical velocity profile.

According to Lliboutry (1981), the integrals $G$ and $F$ can be approximately evaluated:

$$
\begin{aligned}
& G(\bar{z})=\frac{\alpha_{0}+2}{\alpha_{0}+1}\left[1-(1-\bar{z})^{\alpha_{0}+1}\right], \\
& F(\bar{z})=\bar{z}-\frac{(1-\bar{z})}{\alpha_{0}+1}\left[1-(1-\bar{z})^{\alpha_{0}+1}\right]
\end{aligned}
$$

where $\alpha_{0}$ is the apparent exponent in Glen's power law modified in accordance with the non-isothermal conditions in the basal layer. Straightforward estimations by Lliboutry showed that $\alpha_{0}>5-10$. Hence, at least in the upper half of the glacier thickness, the terms $(1-\bar{z})^{\alpha_{0}+1}$ in Equations (3.4) do not exceed $10^{-2}$ and can be omitted. This allows Equation (3.3) to be rewritten in the following form:

$$
\begin{aligned}
u & =\frac{A}{\Delta}\left[1+\frac{\sigma}{\alpha_{0}+1}\right] \\
v & =-w+\partial l / \partial t+u \partial l / \partial s \\
& =\frac{b}{1-\left.c\right|_{h=0}}-\int_{0}^{h} c \omega \mathrm{d} h-\frac{h}{H} \frac{\partial}{\partial s}(H u)
\end{aligned}
$$

where $\Delta=l^{\prime}-z_{0}$ is the thickness of the ice sheet in ice equivalent and $v$ is the downward vertical velocity of the ice sinking into depth relative to the glacier surface.

In central regions of ice sheets, where $A$ and $u$ are rela- tively small, for example in Antarctica at Vostok Station, the accuracy of Equation (3.5) for $v$ is not worse than $10^{-3}$ to $10^{4}$ down to a depth of $h \approx\left(\frac{2}{3}\right) \Delta$.

\section{Macro-strain rates in bubbly ice}

It is now possible to estimate the deviatoric macro-strain rates in the bubbly-ice stratum and to express the tensor $\dot{\mathbf{E}}$ via parameters describing the global flow of the ice sheet and the bubble-compression rate $\omega$. Originally, the coordinate system $h, s$ is curvilinear and not orthogonal. Nevertheless, in each point of the glacier body, we can introduce the local orthogonal (downward and horizontal) directions $h, s$ (see Fig. la) and the transverse one. Let us now designate the normal (diagonal) components of the deviator $\dot{\mathbf{E}}$ along these directions as $q_{1}, q_{2}$ and $q_{3}$, respectively. By definition, from Equations (2.4), (2.7) and (3.5), taking into account that $u$ does not depend on $h$ in the upper part of the ice sheet, we have

$q_{1}=\frac{1}{3} c \omega+\frac{\partial v}{\partial h}, \quad q_{2}=\frac{1}{3} c \omega+\frac{\partial u}{\partial s}, \quad q_{3}=\frac{1}{3} c \omega+\frac{u}{H} \frac{\partial H}{\partial s}$. Then, direct substitution of Equations (3.2) and (3.5) yields $q_{1}=-2 c \omega / 3+q_{1}{ }^{\prime}, \quad q_{2}=c \omega / 3+q_{2}{ }^{\prime}, \quad q_{3}=c \omega / 3+q_{3}{ }^{\prime}$ 
where

$$
\begin{aligned}
q_{1}^{\prime}= & \left(1+\frac{\sigma}{\alpha_{0}+1}\right)\left[\frac{A}{\Delta^{2}} \frac{\partial \Delta}{\partial s}-\frac{1}{\Delta}\left(b-w_{0}-\frac{\partial l^{\prime}}{\partial t}\right)\right], \\
q_{2}^{\prime}= & \left(1+\frac{\sigma}{\alpha_{0}+1}\right) \\
& {\left[\frac{1}{\Delta}\left(b-w_{0}-\frac{\partial l^{\prime}}{\partial t}\right)-\frac{A}{\Delta}\left(\frac{1}{H} \frac{\partial H}{\partial s}+\frac{1}{\Delta} \frac{\partial \Delta}{\partial s}\right)\right], } \\
q_{3}{ }^{\prime}= & \left(1+\frac{\sigma}{\alpha_{0}+1}\right) \frac{A}{\Delta} \frac{1}{H} \frac{\partial H}{\partial s} .
\end{aligned}
$$

Here, $q_{i}{ }^{\prime}, i=1,2,3$, represent the normal deviatoric strain rates of the "external" motion of the ice sheet (see Fig. la and b). Equations (3.6) show that on the macro-scale level the compressive deformation in bubbly ice of a glacier occurs, as illustrated by Figure $1 \mathrm{~b}$ and c, only in the vertical direction.

It should also be noted that the introduced $h, s$ directions are the principal directions of the deformation in the upper part of the ice-flow tube, where the shear stresses and temperatures are comparatively low, to provide noticeable shear strain rates. Then, $q_{1}{ }^{\prime}, q_{2}{ }^{\prime}$ and $q_{3}{ }^{\prime}$ are the only non-zero components of the tensor $\mathbf{E}$ induced by the glacier motion and the corresponding effective strain rate $\epsilon_{a}$ can be determined as:

$$
\epsilon_{a}{ }^{2}=\left(q_{1}{ }^{\prime}\right)^{2}+\left(q_{2}{ }^{\prime}\right)^{2}+q_{1}{ }^{\prime} q_{2}{ }^{\prime} .
$$

Further, it is relevant to define the rate of horizontal icelayer thinning as $\epsilon_{1}=-q_{1}{ }^{\prime}$ and, using Equations (3.6), to write the second invariant $\mathbf{I}_{\mathrm{E}}$ in Equations (2.5) and (2.6) in the form:

$$
\mathbf{I I}_{\mathrm{E}}=\frac{1}{3} c^{2} \omega^{2}+c \omega \epsilon_{1}+\epsilon_{a}{ }^{2} .
$$

As a rule, in the central part of the ice sheet, not far from ice divides, the deformation is close to a simple two-dimensional extension and $\sigma /\left(\alpha_{0}+1\right) \ll 1$. Thus, we have

$$
\epsilon_{1} \approx \epsilon_{a} \approx b / \Delta \text {. }
$$

\section{MATHEMATICAL MODEL OF BUBBLY-ICE DENSIFICATION}

\section{Ice porosity and the rate of bubble compression}

Hereafter we will use subscript "c" to denote the initial conditions $\left(T_{\mathrm{c}}, P_{\mathrm{bc}}, c_{\mathrm{c}}, \ldots\right)$ at the upper boundary of bubbly ice, i.e. at the close-off depth $h_{\mathrm{c}}$ which corresponds to the end of the pore-closure process in the ice sheet. These boundary parameters determine the constant quantity (the mass of air trapped in an ice particle) given by Equation (2.2), from which we obtain

$$
c=\gamma /\left(\gamma+P_{\mathrm{b}}\right), \quad \gamma=P_{\mathrm{s}} \gamma_{s} T / T_{\mathrm{s}}
$$

where

$$
\gamma_{\mathrm{s}}=\frac{c_{\mathrm{c}}}{1-c_{\mathrm{c}}} \frac{P_{\mathrm{bc}} T_{\mathrm{s}}}{P_{\mathrm{s}} T_{\mathrm{c}}}=V \rho_{\mathrm{i}},
$$

whilst $T_{\mathrm{s}}$ and $P_{\mathrm{s}}$ are the standard temperature and pressure (STP: $T_{\mathrm{s}}=273.1 \mathrm{~K}, P_{\mathrm{s}}=0.1013 \mathrm{MPa}$ ), $P_{\mathrm{bc}}$ is defined here as a bubble pressure prevailing at the end of pore closure and $V$ is the air content expressed as a volume (STP) of dry air enclosed in a unit of mass of the ice sample. The dimensionless complex $\gamma_{\mathrm{s}}$ (that must be distinguished from $\gamma$ which is dimensional) is preserved in the "memory" of any bubbly-ice particle in terms of porosity and, thus, has an important role as a paleoclimatic indicator. It may be also directly obtained through air-content measurements.

Mass-balance Equation (2.1) together with Equation (2.4) lead to

$$
\mathrm{d} c / \mathrm{d} t=-c(1-c) \omega
$$

which can be transformed into another equivalent form:

$$
\frac{\mathrm{d}}{\mathrm{d} t} \ln \left(\frac{1-c}{c}\right)=\omega .
$$

Substitution of Equations (4.1) finally gives:

$$
\omega=\frac{T}{P_{\mathrm{b}}} \frac{\mathrm{d}}{\mathrm{d} t}\left(\frac{P_{\mathrm{b}}}{T}\right) .
$$

\section{Absolute load pressure and the pressure in the ice matrix}

Next, we introduce the load pressure

$$
P_{1}=P_{\text {atm }}+g \rho_{\mathrm{i}} h^{\prime}
$$

where $P_{\text {atm }}$ is the atmospheric pressure in the ice equivalent: $h^{\prime}=l^{\prime}-z^{\prime}$.

The averaged pressure in the ice matrix $P_{\mathrm{j}}$ (see Equation (2.1)) and the load pressure $P_{1}$ are not equal. This becomes evident if we write the projection of the momentum-balance Equations (2.1) on the $h$ axis:

$$
\partial P_{\mathrm{i}} / \partial h=\partial T_{1} / \partial h+g \rho_{\mathrm{i}}(1-c)
$$

where $T_{1}$ is the first component in the diagonal of the stress tensor $\mathbf{T}$ for the $h$ direction (additional terms with shear stresses are omitted since they are negligible in the framework of the shallow-ice approximation). The integration of the latter equation taking into account Equation (4.4) yields the following relationship between the two pressures:

$$
P_{\mathrm{i}}=P_{1}+T_{1} \text {. }
$$

Hence, $P_{\mathrm{i}}$ and $P_{1}$ may be equal (which was assumed in previous models) only if $T_{1}$ is negligible. This is not true in general (see the discussion and sensitivity tests below).

\section{Formulation of the model}

Thus, summarizing Equations (2.5)-(2.7), (3.6), (3.7) and Equations (4.1), (4.3)-(4.5), one obtains the model of bubblyice densification:

$$
\begin{aligned}
& \omega= \frac{T}{P_{\mathrm{b}}} \frac{\mathrm{d}}{\mathrm{d} t}\left(\frac{P_{\mathrm{b}}}{T}\right), \quad c=\frac{\gamma_{\mathrm{c}}}{\gamma_{\mathrm{c}}+P_{\mathrm{b}}}, \\
& P_{\mathrm{atm}}+g \rho_{\mathrm{i}} h^{\prime}-P_{\mathrm{b}}=\frac{2}{\sqrt{3}} \int_{2 c \omega / \sqrt{3}}^{2 \omega / \sqrt{3}} \\
& {\left[\eta\left(\xi^{2}+4 \mathbf{I}_{\mathrm{E}}\right)+4 \kappa \mathbf{I}_{\mathrm{E}} \eta^{\prime}\left(\xi^{2}+4 \mathbf{I I}_{\mathrm{E}}\right)\right] \mathrm{d} \xi } \\
&+\frac{4}{3}\left(c \omega+\frac{3}{2} \epsilon_{1}\right) \int_{c}^{1}\left[\eta\left(\frac{4 c^{2} \omega^{2}}{3 \zeta^{2}}+4 \mathbf{I I}_{\mathrm{E}}\right)\right. \\
&\left.+\kappa \frac{4 c^{2} \omega^{2}}{3 \zeta^{2}} \eta^{\prime}\left(\frac{4 c^{2} \omega^{2}}{3 \zeta^{2}}+4 \mathbf{I I}_{\mathrm{E}}\right)\right] \mathrm{d} \zeta ; \\
& \mathbf{I I}_{\mathrm{E}}= \frac{1}{3} c^{2} \omega^{2}+c \omega \epsilon_{1}+\epsilon_{\mathrm{a}}{ }^{2}, \quad \gamma_{\mathrm{c}}=\left.\frac{P_{\mathrm{s}} T}{T_{\mathrm{s}}} \gamma_{\mathrm{s}}\right|_{t=l_{\mathrm{c}}} .
\end{aligned}
$$

The interrelation of the deformation processes occurring in the glacier at different scale levels (schematically depicted in Figure 1) is a highlight of the model. Universal bubble-constriction rate $\omega$ due to the lateral constraints (see Fig. lb) influences deviatoric strain rates $q_{1}, q_{2}$ and $q_{3}$ 
in an ice cell surrounding a bubble (see Equation (3.6) and Fig. lc). As a result, it is equally represented in the second invariant $\mathbf{I I}_{\mathrm{E}}$ of the macro-strain-rate tensor $\dot{\mathbf{E}}$ in Equations (2.5) and (2.6) together with the global strain rates $q_{1}{ }^{\prime}, q_{2}{ }^{\prime}, q_{3}{ }^{\prime}$ (i.e. $\epsilon_{1}$ and $\epsilon_{\mathrm{a}}$ ) of the glacier motion. Consequently, both appear (as $\mathbf{I}_{\mathrm{E}}$ ) in the main Equation (4.6) relating $P_{\mathrm{b}}$ and $\omega$.

For given parameters $\epsilon_{1}, \epsilon_{\mathrm{a}}$ and close-off conditions $h_{\mathrm{c}}, P_{\mathrm{bc}}, \gamma_{\mathrm{s}}$ (i.e. $c_{\mathrm{c}}, T_{\mathrm{c}}$ ), as functions dependent on time $t_{\mathrm{c}}$ and the longitudinal coordinate $s$, the problem in Equations (4.6) is an integro-differential equation with respect to the bubble pressure $P_{\mathrm{b}}$ prevailing in the particle of bubbly ice which was formed at close-off depth at the moment $t=t_{\mathrm{c}}$. The trajectory of the particle: $s=s(t), h=h(t), t>t_{\mathrm{c}}$, is calculated on the basis of Equations (3.5):

$$
\begin{aligned}
\mathrm{d} s / \mathrm{d} t & =u(s, h, t) & \mathrm{d} h / \mathrm{d} t & =v(s, h, t) ; \\
s_{\mid t=t_{\mathrm{c}}} & =s_{\mathrm{c}} & h_{\mid t=t_{\mathrm{c}}} & =h_{\mathrm{c}} .
\end{aligned}
$$

To determine the ice temperature $T$, the energy equation has to be solved simultaneously with Equations (4.6) and (4.7).

\section{MODEL INVESTIGATION}

\section{Ice-flow law}

For practical use of the general model in Equations (4.6) and (4.7), we have to assume a concrete form of the constitutive law in Equation (2.3). Let us accept the polynomial relationship between effective shear strain rate $\dot{e}=\sqrt{\mathbf{I I}_{e}}$ and stress $\tau=\sqrt{\mathbf{I I}_{\tau}}$ as

$$
2 \dot{e}=\tau / M_{1}+\tau^{\alpha} / M_{2} .
$$

Here, $\alpha$ is a creep exponent and $M_{1}$ and $M_{2}$ are the rheological coefficients, which are functions of temperature:

$$
M_{j}=\mu_{j} \exp \left[\frac{Q_{j}}{R_{\mathrm{s}} T_{\mathrm{s}}}\left(\frac{T_{\mathrm{s}}-T}{T}\right)\right], \quad j=1,2
$$

where $\mu_{1}$ and $\mu_{2}$ are the constant factors, $Q_{1}$ and $Q_{2}$ are the apparent activation energies and $R_{\mathrm{S}}$ is the gas constant: $R_{\mathrm{s}}=8.314 \mathrm{~J}(\mathrm{~mol} \cdot \mathrm{K})^{-1}$.

The rheological law given by Equation (5.1) was found to fit closely a wide variety of experimental data (Budd, 1969; Shumskiy, 1969) and the following tentative estimates can be suggested: $\alpha \approx 3.5, Q_{1} \approx Q_{2} \approx 60 \mathrm{~kJ} \mathrm{~mol}^{-1}$, while the factors $\mu_{1}$ and $\mu_{2}$ are of the orders of $1 \mathrm{MPa}$ year and $10^{-2} \mathrm{MPa}^{\alpha}$ year, respectively.

The apparent viscosity $\eta$ in Equation (2.3) is determined as a function of $\lambda=4 \mathrm{II}_{e}$ by

$$
\left[1 / M_{1}+\left(\lambda^{\frac{1}{2}} \eta\right)^{\alpha-1} / M_{2}\right] \eta=1,
$$

which is an immediate consequence of Equation (5.1).

\section{Dimensionless presentation}

At this stage, it is relevant to establish the main dimensionless numbers of the process and to apply similarity theory and scale analysis in order to examine Equations (4.6) and (4.7).

The difference between absolute load pressure and bubble pressure is maximal at the close-off level where it is of the order of $P_{\mathrm{lc}}-P_{\mathrm{atm}}$. The typical value $P^{0}$ of $\left(P_{\mathrm{lc}}-P_{\mathrm{atm}}\right)$ is then to be taken as a scale for stresses in the bubbly-ice stratum. Let us also designate as $b^{0}$ the typical value of the accumulation rate $b$. Then the space $\left(h^{0}\right)$ and time $\left(t^{0}\right)$ scales are written as

$$
h^{0} \approx P^{0} /\left(g \rho_{\mathrm{i}}\right), \quad t^{0} \approx P^{0} /\left(g \rho_{\mathrm{i}} b^{0}\right) .
$$

The corresponding dimensionless variables are distinguished hereafter with super bars:

$$
\begin{gathered}
\bar{h}=\frac{g \rho_{\mathrm{i}} h^{\prime}}{P^{0}}, \bar{t}=\frac{g \rho_{\mathrm{i}} b^{0} t}{P^{0}}, \quad \bar{P}_{\mathrm{b}}=\frac{P_{\mathrm{b}}}{P^{0}}, \bar{\omega}=\frac{\omega P^{0}}{g \rho_{\mathrm{i}} b^{0}}, \bar{T}=\frac{T}{T_{\mathrm{s}}}, \\
\bar{P}_{\mathrm{atm}}=\frac{P_{\mathrm{atm}}}{P^{0}}, \quad \bar{\epsilon}_{1}=\frac{\epsilon_{1} P^{0}}{g \rho_{\mathrm{i}} b^{0}}, \quad \bar{\epsilon}_{\mathrm{a}}=\frac{\epsilon_{\mathrm{a}} P^{0}}{g \rho_{\mathrm{i}} b^{0}}, \quad \bar{\gamma}_{\mathrm{c}}=\left.\frac{P_{\mathrm{s}} T}{P^{0} T_{\mathrm{s}}} \gamma_{\mathrm{s}}\right|_{t=t c} .
\end{gathered}
$$

Consequently, the model in Equation (4.6) can be transformed into the following dimensionless form:

$$
\begin{aligned}
\bar{\omega}= & \frac{\bar{T}}{\bar{P}_{\mathrm{b}}} \frac{\mathrm{d}}{\mathrm{d} t}\left(\frac{\bar{P}_{\mathrm{b}}}{\bar{T}}\right), \quad c=\frac{\bar{\gamma}_{\mathrm{c}}}{\bar{\gamma}_{\mathrm{c}}+\bar{P}_{\mathrm{b}}}, \\
\bar{P}_{\mathrm{atm}}+\bar{h}-\bar{P}_{\mathrm{b}}= & \int_{c \bar{\omega}}^{\omega}\left[\bar{\eta}\left(\xi^{2}+E_{\mathrm{a}}{ }^{2}\right)+\kappa E_{\mathrm{a}}{ }^{2} \bar{\eta}^{\prime}\left(\xi^{2}+E_{\mathrm{a}}{ }^{2}\right)\right] \mathrm{d} \xi \\
& +\left(c \bar{\omega}+\frac{3}{2} \bar{\epsilon}_{1}\right) \int_{c}^{1}\left[\bar{\eta}\left(\frac{c^{2} \bar{\omega}^{2}}{\zeta^{2}}+E_{\mathrm{a}}{ }^{2}\right)\right. \\
& \left.+\kappa \frac{c^{2} \bar{\omega}^{2}}{\zeta^{2}} \bar{\eta}^{\prime}\left(\frac{c^{2} \bar{\omega}^{2}}{\zeta^{2}}+E_{\mathrm{a}}{ }^{2}\right)\right] \mathrm{d} \zeta \\
E_{\mathrm{a}}{ }^{2}= & c^{2} \bar{\omega}^{2}+3 c \bar{\omega} \bar{\epsilon}_{1}+3 \bar{\epsilon}_{\mathrm{a}}{ }^{2}
\end{aligned}
$$

where $\bar{\eta}(\lambda)$ is the solution of the modified Equation (5.3)

$$
\begin{aligned}
{\left[\frac{1}{K_{1}}+\frac{\left(\lambda^{\frac{1}{2}} \bar{\eta}\right)^{\alpha-1}}{K_{2}}\right] \bar{\eta} } & =1, \quad K_{1}=\frac{4 g \rho_{\mathrm{i}} b_{0} M_{1}}{3\left(P_{0}\right)^{2}}, \\
K_{2} & =\frac{g \rho_{\mathrm{i}} b^{0} M_{2}}{\left(P^{0}\right)^{\alpha+1}}\left(\frac{2}{\sqrt{3}}\right)^{\alpha+1} .
\end{aligned}
$$

At a given temperature $\bar{T}$, parameters $K_{1}$ and $K_{2}$ are the main similarity criteria of the densification process. Preliminary estimates show that $K_{1} \sim 0.5-5.0$ and $K_{2} \sim 0.03$ 0.3 at $\alpha=3.5$ for typical ranges of the scales: $P^{0} \sim 0.4-$ $0.7 \mathrm{MPa}, b^{0} \sim 2.0-50 \mathrm{Mg} \mathrm{m}^{-3} \mathrm{a}^{-1}$ and $T_{\mathrm{c}} \sim-50^{\circ}$ to $-20^{\circ} \mathrm{C}$ (see paper II for more detailed data). Another dimensionless parameter $\gamma_{c}$, which is a characteristic (equilibrium) value of the air-volume concentration in the ice, has the order of $10^{-2}$.

In case of the estimate in Equation (3.8), $\bar{\epsilon}_{1}$ and $\bar{\epsilon}_{\mathrm{a}}$ are of the same order as the characteristic space scale divided by the glacier thickness and for $\Delta \sim 1000-3000 \mathrm{~m}$ they are small values:

$$
\bar{\epsilon}_{1} \approx \bar{\epsilon}_{\mathrm{a}} \approx P^{0} /\left(g \rho_{\mathrm{i}} \Delta\right)=h^{0} / \Delta \sim 10^{-2}-10^{-1} .
$$

Since $c$ is also small: $c<0.1$ and $\bar{\omega} \sim 1$, the first term on the righthand side of the integral Equation (5.5) is dominant. This suggests that $P_{\mathrm{i}}$ may be very close to $P_{1}$, whereas the relative external deviatoric macro-strain rates $\left(\bar{\epsilon}_{1}\right.$ and $\left.\bar{\epsilon}_{\mathrm{a}}\right)$ may have only a secondary influence on the bubble-compression rate in central parts of ice sheets. However, a certain (even small) perturbation of the latter equation leads to the corresponding relative change in $\bar{\omega}$ which, in turn, results in a cumulative (integrated) influence on the predictions of the decreasing porosity $c$. The significance of this effect will be demonstrated below in sensitivity tests. It is also obvious that in high-rate-deforming parts of ice sheets, particularly in marginal zones, $\bar{\epsilon}_{1}$ and $\bar{\epsilon}_{\mathrm{a}}$ are not small and may play a primary role in densification processes.

At the same time, a small ratio $h^{0} / \Delta$ is evidence that the relaxation of bubbly ice occurs within a relatively thin layer 
and short time interval. With this in mind, one could examine bubbly-ice densification as a stationary process under constant (mean, present-day) conditions $b, P_{\mathrm{atm}}, h_{\mathrm{c}}, c_{\mathrm{c}} P_{\mathrm{lc}}$, $P_{\mathrm{bc}}$ and $T_{\mathrm{c}}$ (if the typical time-scale of their fluctuations is much larger than $t^{0}$ ) and without regard to possible geographical variations of the close-off characteristics.

Consequently, Equation (4.2) takes the form:

$$
\bar{\nu} \partial c / \partial \bar{h}=-c \bar{\omega}
$$

where $\bar{\nu}=\nu / b^{0}$. In accordance with the definition in Equation (3.6), after differentiation of Equation (3.5) for $\nu$ with respect to $h$, we come to the dimensionless relation

$$
(1-c) \partial \bar{\nu} / \partial \bar{h}=-c \bar{\omega}-\bar{\epsilon}_{1} .
$$

Combining the two latter equations, we obtain

$$
\partial[(1-c) \bar{\nu}] / \partial \bar{h}=-\bar{\epsilon}_{1} .
$$

Hence,

$$
\bar{\nu}=\left(1-\bar{\epsilon}_{1} \bar{h}\right) /(1-c)
$$

and the depth age relationship is given by

$$
\mathrm{d} \bar{t}=\mathrm{d} \bar{h} /\left(1-\bar{\epsilon}_{1} \bar{h}\right) .
$$

Thus, using Equation (5.7) to eliminate the time $\bar{t}$ from Equation (5.5), we find that, for approximately constant temperature $\bar{T} \approx \bar{T}_{\mathrm{c}}$,

$$
\bar{\omega}=\frac{1-\bar{\epsilon}_{1} \bar{h}}{\bar{P}_{\mathrm{b}}} \frac{\mathrm{d} \bar{P}_{\mathrm{b}}}{\mathrm{d} \bar{h}} .
$$

This enables direct calculation of the profiles of $c$ and $\bar{P}_{\mathrm{b}}$ vs depth $\bar{h}$ from Equations (5.5) - (5.8) for the high-rate quasistationary stage of densification.

The corresponding boundary condition at pore close-off can be written as

$$
\left.\bar{P}_{\mathrm{b}}\right|_{\bar{h}=1}=\bar{P}_{\mathrm{bc}} .
$$

\section{Asymptotic phase of the process}

Note that the above model and in particular Equations (5.7)-(5.9) remain valid for any ice particle even if the close-off conditions, at which it was formed, and the accumulation rate have changed in the past, i.e. for ice found at great depths: $\bar{h} \gg 1$. Therefore, the asymptotic behavior of $c$ and $\bar{P}_{\mathrm{b}}$, as $\bar{h} \rightarrow \infty$ i.e. as the age of the particle becomes large), is important and gives a key for reconstructing the variations of the close-off characteristics which are associated with climate, provided that the present-day conditions are comparatively steady.

Indeed, when $c \rightarrow 0$, for $\bar{h} \gg 1$ the bubble pressure $\bar{P}_{\mathrm{b}}$ becomes large in comparison with the integral on the righthand side of Equation (5.5). Hence, we have

$$
\mathrm{d} \bar{P}_{\mathrm{b}} / \mathrm{d} \bar{h} \approx \mathrm{d} \bar{P}_{1} / \mathrm{d} \bar{h}=1
$$

and, using Equation (5.8), we estimate $\bar{\omega}$ as

$$
\bar{\omega} \approx\left(1-\bar{\epsilon}_{1} \bar{h}\right) / \bar{P}_{\mathrm{b}} .
$$

Furthermore, asymptotically, as $c \rightarrow 0$ and $\bar{\omega}$ decreases, Equation (5.5) and (5.6) yield

$$
\begin{gathered}
\bar{P}_{\mathrm{b}}=\bar{P}_{\mathrm{atm}}+\bar{h}-\int_{0}^{\bar{\omega}} \bar{\eta}\left(\xi^{2}+3 \bar{\epsilon}_{\mathrm{a}}^{2}\right) \mathrm{d} \xi-1.5 \bar{\epsilon}_{1} K_{1}, \\
c=\bar{\gamma}_{\mathrm{c}} / \bar{P}_{\mathrm{b}}, \quad \bar{h} \gg 1 .
\end{gathered}
$$

The model in Equations (5.10)-(5.11) is local in time, i.e. $\bar{P}_{\mathrm{b}}$ and $c$ for large depth $\bar{h}$ do not depend on the history of the process. It is also important that at this stage of densification, which we hereafter refer to as an asymptotic phase, the bubble pressure does not depend on the genetic complex $\bar{\gamma}_{\mathrm{s}}\left(\right.$ or $\left.\bar{\gamma}_{\mathrm{c}}\right)$, for which possible variations in accordance with Equations (5.11) are, thus, directly recorded in the porosity profile.

The above theoretical investigation provides a clear general picture of bubbly-ice densification and opens the way to numerical simulation of the process.

\section{COMPUTATIONAL TESTS AND DISCUSSION}

Finite-difference and iterative methods have been used to solve Equations (5.5)-(5.9) numerically. A special interactive computer system ${ }^{*}$ has been developed to study the densification process in glacier ice. It directly calculates porosity and bubble-pressure profiles vs depth $\bar{h}$ for given close-off conditions and pure-ice rheology, and solves the inverse problem of inferring the air content and rheological parameters of pure ice from porosity and/or bubble-pressure measurements. In this section, we discuss the sensitivity of the model results to variations of the different parameters taken in the dimensionless form.

The following values, typical for polar ice in the central parts of Antarctica and Greenland, for instance in Antaretica at Vostok Station, and consistent with the above estimates, have been chosen as basic tentative proxy of the model parameters:

$$
\begin{gathered}
\alpha=3.5, \quad K_{1}=2.5, \quad K_{2}=0.05 \quad \bar{P}_{\mathrm{atm}}=0.1, ; \bar{P}_{\mathrm{bc}}=0.12, \\
c_{\mathrm{c}}=0.075\left(\bar{\gamma}_{\mathrm{c}}=0.01\right), \quad \bar{\epsilon}_{1}=\bar{\epsilon}_{\mathrm{a}}=0.02, \quad \kappa=0.7 . \quad(6.1)
\end{gathered}
$$

Preliminary computations were performed to evaluate the influence of the tuning factor $\kappa$ and the "external" strain rates $\bar{\epsilon}_{\mathrm{a}}, \bar{\epsilon}_{1}$ on the model predictions. As expected, it was found that variations of both $\kappa$, within its uncertainty range from 0.4 to 1.0 , and $\bar{\epsilon}_{\mathrm{a}}, \bar{\epsilon}_{1}$, from 0 to 0.04 (a plausible upper bound for the central parts of the ice sheets), do not change the solution notably. The maximum deviation of the porosity $c$ and the bubble pressure $\bar{P}_{\mathrm{b}}$ from the basic profiles was observed to be within $\pm 1.5-2 \%$. Subsequently, these parameters $\left(\kappa, \bar{\epsilon}_{\mathrm{a}}, \bar{\epsilon}_{1}\right)$ were fixed in accordance with Equations (6.1) and remained unchanged for all the following computational runs.

\section{Sensitivity to the rheological parameters of pure ice}

The main series of the tests focused on the model sensitivity to the rheological parameters $M_{1}$ and $M_{2}$ (i.e. to variations of the criteria $K_{1}$ and $K_{2}$. The two terms of the polynomial law in Equation (5.1) obviously represent linear and power asymptotes for low and high stresses $\tau$, respectively. Since the micro-scale stresses associated with bubbly-ice densification fall mostly in the transition zone between linear and power rheolgical behaviors, both of the criteria are important. The transition zone is centered at $\tau \approx\left(M_{1} / M_{2}\right)^{1 /(\alpha-1)}$ and for the corresponding range of stresses Equation (5.1) can be rewritten in the following normalized form:

$$
\begin{gathered}
\overline{\dot{e}}=\bar{\tau}+\bar{\tau}^{\alpha}, \\
\overline{\dot{e}}=2 M_{1}\left(M_{1} / M_{2}\right)^{1 /(\alpha-1)} \dot{e}, \quad \bar{\tau}=\left(M_{1} / M_{2}\right)^{1 /(\alpha-1)} \tau .2
\end{gathered}
$$

Relation (6.2) is represented in logarithmic scales in Figure $2 \mathrm{a}$ as curve 1 (the dashed lines show its asymptotes). Let

\footnotetext{
* The code of the system for IBM-PC compatible computers is available from the authors on request.
} 

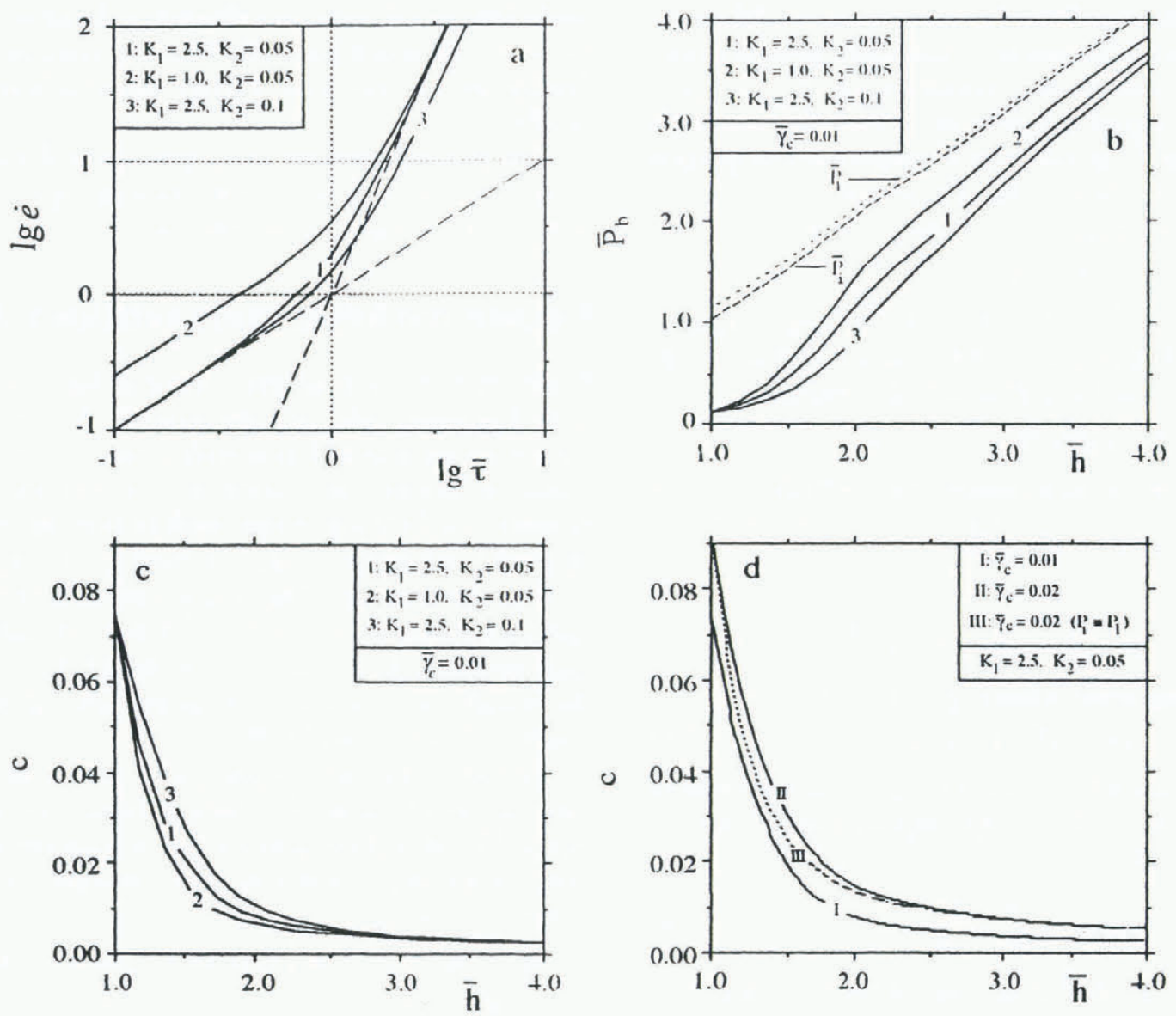

Fig. 2. Influence of pure-ice rheology and boundary conditions at pore close-off on ice-porosity and bubble-pressure profiles in ice sheets as revealed by computational tests. (a) Polynomial rheological law of ice in the normalized form. The curves $1-3$ correspond to the various parameters $K_{1}$ and $K_{2}$ (indicated on the figure). The dashed lines are asymptotes of curve 1 . ( $b$ ) Relative bubble pressure $\bar{P}_{\mathrm{b}}$ vs normalized depth $\bar{h}$. The profiles $1-3$ are computed with the same pairs of the parameters $K_{1}$ and $K_{2}$ as in $(a)$, and under fixed close-off conditions $\left(\bar{\gamma}_{\mathrm{c}}=0.01\right)$. The dotted and dashed lines represent absolute load pressure $\bar{P}_{1}$ and the pressure $\bar{P}_{\mathrm{i}}$ in the ice matrix, respectively. (c) Porosity $(c)$-depth $(\bar{h})$ profiles $1-3$ calculated with the same model parameters as referred to for identically numerated $\bar{P}_{\mathrm{b}}-\bar{h}$ profiles in $(b)$. (d) Porosity profiles I-III calculated with fixed ice rheology corresponding to the basic curve 1 in (a), and for various $\bar{\gamma}_{c}$ (as indicated on the figure). The profile III (dotted line) is simulated with the same parameters as the curve II but $\bar{P}_{\mathrm{i}}$ is presumed to be identical to $\overline{\mathrm{P}}_{1}$.

us assume now that curve 1 corresponds to the basic values: $K_{1}=2.5, K_{2}=0.05$. Then, if the criteria $K_{1}$ and $K_{2}$ are alternately changed the initial curve is modified, for example: at $K_{1}=1.0$ and $K_{2}=0.05$ we obtain curve 2 , while at $K_{1}=$ 2.5 and $K_{2}=0.1$ we get curve 3 in Figure 2a. Thus, Figure $2 \mathrm{a}$ illustrates a selective response of the polynomial rheological curve at low (high) stresses to a change mostly of the criterion $K_{1}\left(K_{2}\right)$. When using the ice-flow law given by curves $1-3$ in Figure 2 a to simulate the densification process under fixed close-off conditions $\left(\bar{\gamma}_{c}=0.01\right)$, we obtain the corresponding profiles of bubble pressure $\bar{P}_{\mathrm{b}}$ (curves $1-3$ in Figure 2b) and ice porosity $c$ (curves 1-3 in Figure 2c) vs depth $\bar{h}$.

The difference between curves 1 and 2 in Figure $2 b$ illustrates the fact that the bubble-pressure profile is significantly influenced by variations of the rheological parameter $M_{1}$. The maximal perturbations are observed, as expected, at large depth, i.e. in the low-stress range. On the other hand, when $M_{2}$ is changed (compare curves 1 and 3 in Figure $2 \mathrm{~b}$ ), only the upper part of the bubble-pressure profile is affected significantly in the zone where the effective stresses are relatively high. Thus, the bubble pressure is closely and selectively linked to the rheological properties of pure ice. On the contrary, no significant change in the simulated porosity profile is observed at great depth, even if the "linear" viscosity $M_{1}$ is changed, while the upper part of the profile equally depends on both of the rheological parameters $M_{1}$ and $M_{2}$ (see the curves 1-3 in Figure 2c). Therefore, the impacts of the factors $M_{1}$ and $M_{2}$ on density relaxation are practically indistinguishable.

\section{Sensitivity to the pore close-off conditions}

According to Equation (4.1), when $P_{\mathrm{b}} \gg \gamma_{\mathrm{c}}$, the porosity $c$ is approximately proportional to the air content $V$. Therefore, $c$ keeps "memory" about past close-off conditions. Indeed, a high sensitivty of the ice-porosity profile to realistic variations (see, for instance, paper II) of the genetic complex $\bar{\gamma}_{\mathrm{c}}$ is evident when comparing the profiles $\mathrm{I}\left(\bar{\gamma}_{\mathrm{c}}=0.01\right)$ and II $\left(\bar{\gamma}_{c}=0.02\right)$ in Figure $2 \mathrm{~d}$ (both of the profiles were simulated with the same rheological parameters as those used for curves 1 in Figure $2 b$ and c). On the other hand, numerical experiments show that after pore close-off the bubble pressure $\bar{P}_{\mathrm{b}}$ very quickly becomes insensitive to plausible changes in $\bar{\gamma}_{c}$. This is in agreement with approximate Equations (5.10) and (5.11). All of the computational tests have confirmed that the asymptotic phase starts from $\bar{h} \approx 3.0$.

\section{Significance of the model improvements}

Summarizing the results presented above, we come to the 
following conclusions: (i) the stationary model is sufficient for interpreting the experimental data on bubbly-ice densification; (ii) both ice-porosity and bubble-pressure experimental profiles are necessary to obtain an adequate solution of the inverse problem of inferring rheological parameters of pure ice; (iii) the porosity profile can also be employed to deduce past variations of the pore close-off conditions expressed in terms of the dimensionless complex $\gamma_{\mathrm{s}}$ which is proportional to air content in ice. The improvements made in the present consideration with respect to the earlier studies by Salamatin and Lipenkov (1993), such as incorporating the generalized rheological model of bubbly ice and taking into account the difference between absolute load pressure and averaged pressure in the ice matrix, are important for both inverse and forward problems. The significance of the latter factor for predicting the ice-porosity profile in the relaxation phase of bubbly-ice densification has already been discussed in section 5 and is demonstrated in Figure 2d. One can see here that the discrepancy between simulated profiles II and III obtained with the same initial data, except that $P_{\mathrm{i}} \equiv P_{1}$ for profile III, may be similar to the impact of the principal model parameters: $K_{1}, K_{2}$ and $\bar{\gamma}_{\mathrm{c}}$. The effect of using constitutive relations more sophisticated than the power-creep law is also shown to be substantial for simulating the densification process with respect to bubble-pressure relaxation. However, this innovation does not significantly influence the results while modeling the post-drilling relaxation of ice density (Lipenkov and Salamatin, 1989), since the latter process takes place at a relatively high bubble-ice pressure drop, i.e. within the stress range where the power-law creep of ice is valid.

\section{CONGLUSION}

A generalized model of bubbly-ice densification beneath the close-off depth has been developed and investigated on the basis of similiarity theory and scale analysis. The interaction between the universal compression of bubbles and deviatoric strains in a non-linear viscous ice matrix is the principal peculiarity of the deformation process in the upper stratum of ice sheets. Due to this, the rate of bubblyice densification may be considerably influenced by the global flow of the glacier but mainly in its hydrodynamically active regions.

Two phases of the densification have been identified. The first is a relatively short phase of densification governed by the initial difference between the absolute load pressure of ice sediments and the average pressure in air bubbles at the pore close-off depth. This phase takes place within the depth interval $1.0<\bar{h} \leq 3.0$, where $\bar{h}$ is the ice-equivalent depth normalized so that $\bar{h}=1$ at close-off. The difference between the absolute load pressure and the averaged pressure in the ice matrix is important especially when modeling porosity variations within this depth range. The second, the asymptotic phase of bubbly-ice densification, takes place at $\bar{h}>3.0$, under minimal bubble-ice pressure drop which is governed by the present-day accumulation rate. The rate of bubble compression at this stage is not sensitive to past changes in the pore close-off conditions and the bubble pressure does not depend on the air content in ice. On the other hand, the ice-porosity profile is found to be responsive to air-content variations.

Both ice-porosity and bubble-pressure profiles in ice sheets are sensitive to the rheological parameters of pure ice but only the bubble-pressure profile distinguishes the rheological properties at low and high stresses. Finally, the stationary model of bubbly-ice densification appears to be sufficient for interpreting the experimental data, provided that the conditions at the pore close-off depth are relatively steady during the typical duration of the process. In the accompanying paper (paper II), we shall formulate the inverse problem and use the model to deduce rheological properties of pure ice as well as to estimate the air content from porosity and bubble-pressure profiles measured in ice sheets.

\section{ACKNOWLEDGEMENTS}

The authors are grateful to their colleagues from Kazan State University: V. A. Chugunov, A. G. Yegorov, L. D. Eskin as well as to T. Hondoh from the Institute of Low Temperature Science (Sapporo) and J. Meyssonnier from the Laboratoire de Glaciologie et Géophysique de l'Environnement (L.G.G.E.), Grenoble, for helpful and stimulating discussions. Special thanks are addressed to P. Martinerie (L.G.G.E.) who thoroughly read the draft and suggested many constructive improvements. We also highly appreciate the very useful comments by $\mathrm{K}$. Hutter Institut für Mechanik, Darmstadt) taken into account in the final version of the paper.

This research was supported in Russia by the Russian Federation State Committee for Science and Technologies and the Russian Basic Research Foundation, and in France by the Programme National d'Etudes de la Dynamique du Climat (C.N.R.S), the Département Sciences Pour l'Ingénieur du C.N.R.S. and Le Congrès de Mécanique à Grenoble.

\section{REFERENCES}

Bader, H. 1965. Theory of densification of dry, bubbly glacier ice. CRREL Res. Rep. 141.

Bensoussan, A., J. -L. Lions and G. Papanicolaou. 1978. Asymptotic analysis for periodic structures. Amsterdam, North-Holland.

Budd, W. 1969. The dynamics of ice masses. A.NARE Sci. Rep., Ser. A (IV). Glaciology 108

Glen, J. W. 1955. The creep of polycrystalline ice. Proc. R. Soc. London, Ser. A $228+1175,519-538$.

Gow, A.J. 1968. Bubbles and bubble pressures in Antarctic glacier ice. f. Glaciol., $7(50), 167-182$.

Langway, C. C., Jr. 1958. Bubble pressures in Greenland glacier ice. International Association of Scientific Hydrology Publication 47 (Symposium at Chamonix 1958 - Physics of the Movement of the Ice), 336-349.

Lipenkov, V.Ya. 1989. Obrazovanive i razlozheniye gidratov vozdukha v lednikovom l'du [Formation and decomposition of air hydrates in glacier ice]. Mater. Glyatsiol. Issled. 65, 58-64.

Lipenkov, V. Ya. and A. N. Salamatin. 1989. Relaksatsionnoye rasshireniye ledyanogo kerna iz burovoy skvazhini na st. Vostok [Volume relaxation of the ice core from the bore hole at Vostok Station]. Antarktika 28, 59-72.

Lipenkov, V.Ya., N. I. Barkov, P. Duval and P. Pimienta. 1989a. Crystalline texture of the $2083 \mathrm{~m}$ ice core at Vostok Station, Antarctica. F. Glaciol. 35 (121), 392-398.

Lipenkov, V.Ya., A. N. Salamatin and Yu. A. Grigor'yeva. 1989b. Matematicheskaya model' i chislennoye issledovaniye protsessa uplotneniya lednikovogo l'da [Mathematical model and numerical studies of glacier ice densification process]. Mater. Glyatsiol. Issled. 65, 49-58.

Lipenkov, V. Ya., A. N. Salamatin and P. Duval. 1997. Bubbly-ice densification in ice sheets: II. Applications. f. Glaciol., 43 145),397-407.

Lliboutry, L. 1981. A critical review of analytical approximate solutions for steady state velocities and temperatures in cold ice-sheets. Z. Gletscherkd. Glazialgeol., 15 (2), 1979, 135-148.

Miller, S. L. 1969. Clathrate hydrates of air in Antarctic ice. Science, $\mathbf{1 6 5}(3892), 489-490$.

Nakawo, M. and H. Narita. 1985. Density profile of a $413.5 \mathrm{~m}$ deep fresh core 
recovered at Mizuho Station, East Antarctica. Mem. Nall. Inst. Polar Res., Spec. Issue 39, 141-156.

Pimienta, P. 1987. Étude du comportement mécanique des glaces polycrystallines aux faibles contraintes: application aux glaces des calottes polaires. (Thèse de Doctorat, Université Scientifique, Technologique et Médicale de Grenoble.) (LGGE Publication 544.

Pimienta, P. and P. Duval. 1989. Rheology of polar glacier ice. (Abstract.) Ann. Glaciol., 12, $206-207$.

Salamatin, A.N. 1991. Ice sheet modelling taking account of glacier ice compressibility: International Association of Hydrological Sciences Publication 208 (Symposium at St Petersburg 1990 - Glaciers-Ocean-Atmosphere Interactions), $183-192$.
Salamatin, A.N. and P. Duval. 1997. Creep flow and pressure relaxation in bubbly medium. Int. J. Solids Struct., 34 (1), $61-78$.

Salamatin, A. N. and V.Ya. Lipenkov: 1993. Theoretical studies on densification and relaxation of bubbly glacier ice. Antarct. Rec., 37 (3), 265-276.

Salamatin, A. N., V. Ya. Lipenkov, K. Ye. Smirnov and Yu. V. Zhilova. 1985. Plotnost' lednikovogo l'da i yego reologicheskiye svoystva [The density of glacier ice and its rheological properties]. Antarktika 24, 94-106.

Shumskiy, P. A. 1969. Gidrologiya sushi. Glyatsiologiya. Dinamicheskaya glyatsiologiya [Land hydrology. Glaciology. Dynamic glaciology]. Itogi Nauki, Ser. Geografiya 1, 1968.

Wilkinson, D. S. and M. F. Ashby. 1975. Pressure sintering by power law creep. Acta Metall., 23 (11), 1277-1285.

MS received 11 March 1996 and accepted in revised form 6 February 1997 